\title{
BioMAX, a macromolecular crystallography facility at MAX IV Ana Gonzalez ${ }^{1}$ \\ ${ }^{1}$ MAXIV \\ ana.gonzalez@maxiv.lu.se
}

BioMAX is the first operational Macromolecular Crystallography (MX) beamline at the MAX IV facility.[1] The design goal was to create a stable and reliable multipurpose beamline. The beamline optics consist of a double crystal $\mathrm{Si}(111)$ monochromator that provides an energy range between 5 and $25 \mathrm{keV}$ and a pair of $\mathrm{KB}$ mirrors; the beam can be focused down to $20 \times 5 \mu \mathrm{m} 2 \mathrm{FWHM}$ at the sample position with a photon flux of about $5 \times 1012$ photons $/ \mathrm{s}$ at a ring current of $250 \mathrm{~mA}$ and an energy of $13 \mathrm{keV}$. Changes of energy and beam focus (up to $100 \mathrm{x} 100$ $\mu \mathrm{m} 2$ ) are automated. The experimental hutch is equipped with a MD3 microdiffractometer, an IRELEC Isara sample changers and a 16M Eiger detector. MXCuBE3 is used for user beamline control and data collection.[2]

A wide range of MX experimental techniques are available at the beamline, from standard oscillation data collection at cryo-temperatures, optimized SAD and MAD experiments, humidity-controlled room temperature data collection and Serial Crystallography experiments, both fixed target and injector based.[3] A fragment-based drug discovery facility (FragMAX) is also based a BioMAX.[4] Conventional experiments at cryotemperatures can be done fully remotely.

References

[1] Ursby et al. BioMAX - the First Macromolecular Crystallography Beamline at MAX IV Laboratory. J. Synchrotron Radiat. 2020, 27 (5), 1415-1429. DOI: 10.1107/s1600577520008723

[2] Mueller et al. MXCuBE3: A New Era of MX-Beamline Control Begins. Synchrotron Radiat. News 2017, 30 (1), 22-27. DOI: $10.1080 / 08940886.2017 .1267564$

[3] Shilova et al. Current Status and Future Opportunities for Serial Crystallography at MAX IV Laboratory. J. Synchrotron Radiat. 2020, 27 (5), 1095-1102. DOI: 10.1107/S1600577520008735

[4] Lima et al. FragMAX: The Fragment-Screening Platform at the MAX IV Laboratory. Acta Crystallogr. Sect. D, Struct. Biol. 2020, 76 (8), 771-777. DOI: 10.1107/S205979832000889X, 